\title{
Spectroscopic and photometric studies of white dwarfs in the Hyades
}

\author{
P.-E. Tremblay ${ }^{1}$, E. Schilbach ${ }^{2}$, S. Röser ${ }^{2}$, S. Jordan ${ }^{2}$, H.-G. Ludwig ${ }^{1}$, and B. Goldman ${ }^{3}$ \\ 1 Zentrum für Astronomie der Universität Heidelberg, Landessternwarte, Königstuhl 12, 69117 Heidelberg, Germany \\ e-mail: [ptremblay;hludwig]@lsw.uni-heidelberg.de \\ 2 Zentrum für Astronomie der Universität Heidelberg, Astronomisches Rechen-Institut, Mönchhofstrasse 12-14, 69120 Heidelberg, \\ Germany \\ e-mail: [elena;roeser;jordan]@ari.uni-heidelberg.de \\ ${ }^{3}$ Max-Planck-Institut für Astronomy, Königstuhl 17, 69117 Heidelberg, Germany \\ e-mail: goldman@mpia.de
}

Received 19 July 2012 / Accepted 18 September 2012

\section{ABSTRACT}

\begin{abstract}
The Hyades cluster is known to harbour ten so-called classical white dwarf members. Numerous studies through the years have predicted that more than twice this amount of degenerate stars should be associated with the cluster. Using the PPMXL Catalog of proper motions and positions, a recent study proposed 17 new white dwarf candidates. We review the membership of these candidates by using published spectroscopic and photometric observations, as well as by simulating the contamination from field white dwarfs. In addition to the ten classical Hyades white dwarfs, we find six white dwarfs that may be of Hyades origin and three more objects that have an uncertain membership status due to their unknown or imprecise atmospheric parameters. Among those, two to three are expected as field stars contamination. Accurate radial velocity measurements will confirm or reject the candidates. One consequence is that the longstanding problem that no white dwarf older than $\sim 340 \mathrm{Myr}$ appears to be associated with the cluster remains unsolved.
\end{abstract}

Key words. stars: atmospheres - white dwarfs - open clusters and associations: individual: Hyades - stars: kinematics and dynamics

\section{Introduction}

The Hyades cluster, with a centre-of-mass distance of $46.45 \pm$ $0.5 \mathrm{pc}$ (van Leeuwen 2009) and an estimated age of $625 \pm$ $50 \mathrm{Myr}$, derived from the helium abundance and isochrone modelling (Perryman et al. 1998), is certainly one of the most studied open clusters. Recently, Röser et al. (2011, hereafter Paper I) identified 724 probable main-sequence members within a radius of $30 \mathrm{pc}$ from the cluster centre. Their sample is based on a kinematic selection from the PPMXL Catalog (Röser et al. 2010) with the convergent point method, further constrained by photometric data. They determined individual masses for their candidates and, among others, studied the mass distribution as a function of the distance to the centre. It was confirmed that the present day tidal radius of the cluster is $\sim 9 \mathrm{pc}$, and that this value would only change by a few percent under different assumptions on the fraction of binaries, a quantity that is currently not well constrained.

In this work, we restrict our study to Hyades white dwarfs, even for which the literature is extensive. It was already concluded long ago that old white dwarfs with an age greater than $~ 300$ Myr were missing from the cluster (Tinsley 1974; van den Heuvel 1975), and that they might have evaporated (Pels et al. 1975; Weidemann 1977). Weidemann et al. (1992) and Eggen (1993) further constrained this scenario by suggesting that old evaporated Hyades white dwarfs form long tidal tails for which the density is much lower than that of field stars. To this day, it is still the case that only 10 constituents are confirmed, and $\mathrm{N}$-body simulations systematically predict that at least two times more white dwarfs were formed in the Hyades (Portegies Zwart et al. 2001; Ernst et al. 2011). The idea that many remnants evaporated from the cluster remains a likely scenario, which is further suggested by the fact that Paper I found prominent tidal tails for main-sequence stars, with almost as many members currently leaving the cluster than members bound to the cluster. However, it is also possible that the missing white dwarfs were simply never formed because of a very steep initial mass function (Böhm-Vitense 2007) or that they are hidden in binaries (Portegies Zwart et al. 2001). Williams (2004) tried to bring the observed number of white dwarfs in the Hyades into agreement with calculated numbers by varying the slope of the IMF, the binary fraction, the binary mass ratio and the effect of dynamical evolution. He concludes that, from simulations alone, it is not possible to differentiate between the above scenarios. A deficiency of white dwarfs has also been observed in several other open clusters (see, e.g., Richer et al. 1998; Kalirai et al. 2001b; Williams \& Bolte 2007), although there are also many instances where the observed and predicted numbers of degenerate objects are in agreement (Kalirai et al. 2001a, 2003; Rubin et al. 2008).

In a recent analysis, Schilbach \& Röser (2012, hereafter Paper II) extended the systematic search of Hyades members to white dwarfs up to $40 \mathrm{pc}$ from the cluster centre. The authors did recover all 10 classical components, and further identified 17 white dwarfs as new candidates, all of them outside of the tidal radius. If these degenerate stars are confirmed as members, they could potentially explain the discrepancy between the observed and predicted number of associated objects, and also confirm that white dwarfs are slowly leaving the cluster.

The 10 classical Hyades white dwarfs are certainly some of the most well known and observed hot remnants. Given that new 
atmospheric parameters have recently been published for most of these objects based on spectroscopic analyses (Gianninas et al. 2011; Bergeron et al. 2011) relying on improved model atmospheres (Tremblay \& Bergeron 2009), it is certainly pertinent to review their properties as Hyades representatives. Furthermore, most of the 17 new candidates identified in Paper II also have well determined atmospheric parameters, from spectroscopic or photometric analyses. Consequently, the next step to constrain their membership is to compare quantities derived from kinematic studies, e.g. kinematic distances, with those derived from their atmospheric properties, which includes the socalled spectroscopic distance, based on predicted luminosities (Holberg et al. 2008). The spectroscopic distances together with the proper motions can be used to update the space velocities for candidates, i.e. the residual $2 \mathrm{D}$ velocity with respect to the Hyades bulk motion, which is important to understand the structure of the cluster.

This work is structured as follow. In Sect. 2, we describe the Hyades white dwarfs sample of Paper II and Sect. 3 follows with a description of analyses of their spectra and photometry. Based on their derived atmospheric parameters, we compute, in Sect. 4, spectroscopic distances for all aspirant Hyades remnants. In Sect. 5, we derive improved residual velocities, and try to understand the global status of new candidates by simulating the field white dwarfs contamination. Then, Sect. 6 continues with a discussion on the membership of new candidates and the implications on the Hyades evolution. The summary is presented in Sect. 7.

\section{Kinematic and photometric Hyades candidates}

The starting point of our analysis are the Hyades white dwarf candidates identified in Paper II. The selection process is explained in details in Paper I and II. In brief, the primary data of observations are the proper motions and positions in the PPMXL Catalog. The PPMXL is an all-sky survey with a limiting magnitude of $V \sim 20$ including about 900 million objects. The PPMXL data was cross-matched and combined with the UCAC3 (Zacharias et al. 2010) and the CMC14 (Copenhagen Univ. Obs. et al. 2006), and referred as a whole as the CarlsbergUCAC (CU) subset. Along with the kinematics, this data set also includes 2MASS $J H K_{\mathrm{S}}$ and $\mathrm{CMC} 14 r^{\prime}$ magnitudes.

Candidates are first selected using the convergent point method (Paper I). More precisely, in the case of white dwarf candidates, an upper limit of $5 \mathrm{~km} \mathrm{~s}^{-1}$ is allowed for the difference between their tangential motion and the bulk motion of the Hyades. The convergent point method then predicts, for each possible member, a radial velocity and a distance, hereafter named the kinematic distance $\left(d_{\text {kin }}\right)$, based only on the position $(\alpha, \delta)$ and proper motion $\left(\mu_{\alpha}, \mu_{\delta}\right)$. To avoid contamination by field objects, only the new candidates with a $d_{\text {kin }}$ prediction that put them closer than $40 \mathrm{pc}$ from the cluster centre are kept.

The second step used in Paper II to construct the Hyades sample was to cross-match all kinematically suited candidates with white dwarf catalogues, such as the online version of the Villanova White Dwarf Catalog ${ }^{1}$ (McCook \& Sion 1999). From this procedure, the authors identified a total of 37 white dwarfs as kinematic candidates. We note that at this point, a fair number of these objects could be background objects with proper motions that mimic the Hyades motion. Therefore, the final step was to examine the available photometric data of the candidates.

\footnotetext{
1 http://WWW . astronomy . villanova. edu/WDCatalog/index. html
}
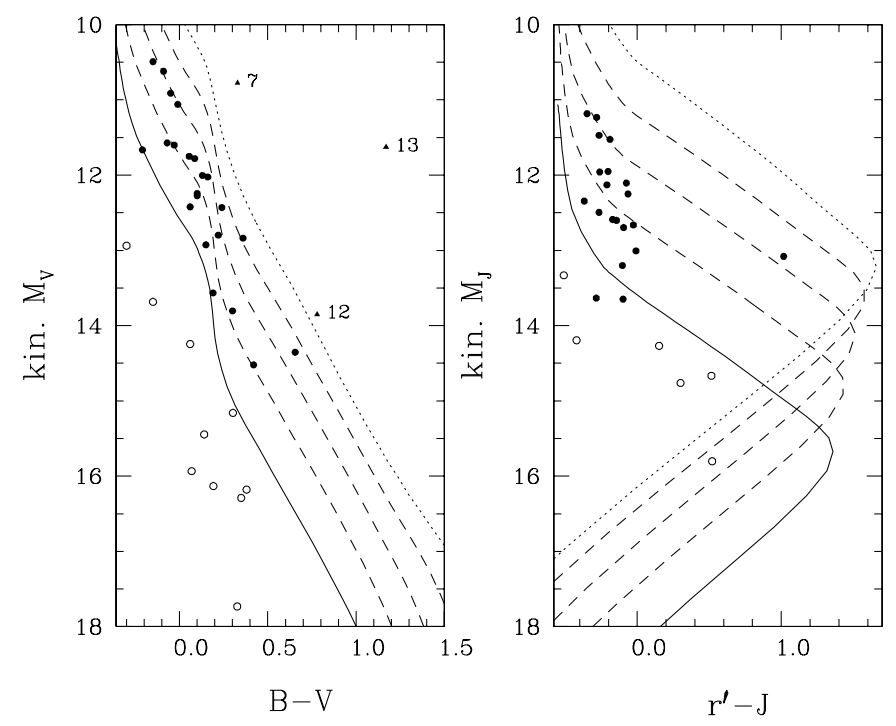

Fig. 1. Kinematic absolute magnitude versus color diagrams for Hyades white dwarf candidates (filled circles) and probable non-Hyades members (open circles) identified in Paper II. Binaries are marked as filled triangles and by their numbers from Table 1, although in most cases, they are too bright for the range of absolute magnitudes shown in the figure. Theoretical cooling sequences are shown from $\log g=7.0$ (dotted line) to $\log g=9.0$ (solid line) in steps of $0.5 \mathrm{dex}$.

These white dwarfs are unfortunately at the detection limit of the 2MASS and CMC14 survey. Even if $J H K_{\mathrm{s}}$ and $r^{\prime}$ magnitudes are available for most candidates, a high scatter was found in the $J-K_{\mathrm{S}}$ colors, which is consistent with the conclusion of Tremblay \& Bergeron (2007) that one should be cautious with $K_{\mathrm{s}}$ magnitudes near the faint detection limit of the survey. Therefore, it was opted to include in the analysis $B$ and $V$ magnitudes from the literature even if the accuracy of the available $B V$ was far from being uniform and varied from several millimag (photoelectric measurements) to several tenths of a mag (photographic measurements).

Using the kinematic distance and an observed magnitude, a "kinematic" absolute magnitude (kin. $M$ ) can be computed. In Fig. 1 we reproduce the kin. $M_{V}$ vs. $B-V$ and kin. $M_{J}$ vs. $r^{\prime}-J$ diagrams made in Paper II, but now compared with theoretical white dwarf cooling sequences (Wood 1995; Fontaine et al. 2001). True Hyades members are expected to follow the theoretical sequences. On the contrary, background and foreground stars are expected to be shifted vertically in relation to the theoretical sequences, because their estimated $d_{\text {kin }}$ differs from the true distance. We can see from Fig. 1 that the 10 white dwarfs identified as probable non-members in Paper II (open circles) lie below the theoretical cooling sequences. They would have to be super massive $(\log g>9.0)$ to be cluster members. Instead, they are much more likely distant objects mimicking the Hyades proper motion.

All 10 of the so-called classical Hyades members were recovered in Paper II. Removing 10 white dwarfs identified as probable non-members, as discussed above, there remain 17 new candidates, all lying outside of the tidal radius of $\sim 9 \mathrm{pc}$. However, five of the remaining candidates are more than $20 \mathrm{pc}$ away from the cluster centre in the $Z$ direction (perpendicular to the galactic plane) and main-sequence stars with known radial velocities in these regions have been ruled out as members (Paper I). We note that three of the classical members and two of the new candidates are unresolved binaries with a main-sequence companion. 
Table 1. Atmospheric parameters of Hyades white dwarf candidates.

\begin{tabular}{|c|c|c|c|c|c|c|c|c|c|c|}
\hline Star No. & WD name & Other name & SpT & $\begin{array}{l}T_{\text {eff }} \\
(\mathrm{K})\end{array}$ & $\begin{array}{c}\log g \\
\left(\mathrm{~cm} \mathrm{~s}^{-2}\right)\end{array}$ & $M / M_{\odot}$ & $\begin{array}{c}M_{V} \\
(\mathrm{mag})\end{array}$ & $\begin{array}{c}\log \tau \\
(\mathrm{yr})\end{array}$ & $\begin{array}{l}\text { dist } \\
\text { (pc) }\end{array}$ & Ref. \\
\hline 1 & $0347+171$ & V471 Tau & $\mathrm{DA}+\mathrm{dK}$ & $34100(100)$ & $8.25(0.05)$ & $0.84(0.04)$ & $10.20(0.12)$ & $7.07(0.13)$ & - & 1 \\
\hline 2 & $0352+096$ & HZ 4 & DA & $14670(380)$ & $8.30(0.05)$ & $0.80(0.03)$ & $11.76(0.09)$ & $8.53(0.05)$ & 36 & 2 \\
\hline 3 & $0406+169$ & LB 227 & DA & $15810(290)$ & $8.38(0.05)$ & $0.85(0.03)$ & $11.76(0.09)$ & $8.50(0.04)$ & 52 & 2 \\
\hline 4 & $0418+137$ & HD 27483 & $\mathrm{DA}+\mathrm{dF}$ & $21410(210)$ & $8.00(0.50)$ & 0.63 & 10.65 & 7.70 & - & 3 \\
\hline 5 & $0421+162$ & LP 415-46 & DA & $20010(320)$ & $8.13(0.05)$ & $0.70(0.03)$ & $10.96(0.08)$ & $7.97(0.06)$ & 47 & 2 \\
\hline 6 & $0425+168$ & LP 415-415 & DA & $25130(380)$ & $8.12(0.05)$ & $0.71(0.03)$ & $10.53(0.08)$ & $7.49(0.08)$ & 50 & 2 \\
\hline 7 & $0429+176$ & HZ 9 & $\mathrm{DA}+\mathrm{dM}$ & $17620(350)$ & $8.02(0.06)$ & $0.63(0.04)$ & $11.02(0.09)$ & $8.08(0.06)$ & 39 & $2 \mathrm{~A}$ \\
\hline 8 & $0431+126$ & HZ 7 & DA & $21890(350)$ & $8.11(0.05)$ & $0.69(0.03)$ & $10.78(0.08)$ & $7.78(0.07)$ & 49 & 2 \\
\hline 9 & $0437+138$ & LP 475-242 & DBA & $15120(360)$ & $8.25(0.09)$ & $0.74(0.06)$ & $11.65(0.15)$ & $8.47(0.07)$ & 45 & 4 \\
\hline 10 & $0438+108$ & HZ 14 & DA & $27540(400)$ & $8.15(0.05)$ & $0.73(0.03)$ & $10.40(0.08)$ & $7.30(0.09)$ & 49 & 2 \\
\hline 11 & $0120-024$ & LP 587-53 & $\overline{D A}$ & $5880(80)$ & $8.12(0.16)$ & $0.66(0.10)$ & $14.53(0.24)$ & $9.51(0.15)$ & - & 5 \\
\hline 14 & $0230+343$ & GD 30 & DA & $15270(250)$ & $7.98(0.05)$ & $0.60(0.03)$ & $11.21(0.08)$ & $8.26(0.04)$ & 91 & 2 \\
\hline 15 & - & LP 246-14 & - & 9680 (2600) & $8.00(0.50)$ & 0.60 & 12.31 & 8.82 & - & 6 \\
\hline 16 & $0259+378$ & GD 38 & DA & 32040 (470) & $7.84(0.05)$ & $0.57(0.03)$ & 9.59 (0.09) & $6.89(0.02)$ & 174 & 2 \\
\hline 17 & 0 & Gl & DA & 0) & 7.89 & 0.56 & 10.74 & 7.86 & 105 & 2 \\
\hline 18 & 35 & LP 6 & DA & 130 & 8.0 & 0.6 & & 8.51 & 52 & 2 \\
\hline 19 & $0348+339$ & GD & $\mathrm{D}$ & 148 & 8 & 0.80 & 11. & 8.52 & 49 & 2 \\
\hline 20 & - & 040 & D & 14 & 8.2 & 0.76 & 11 & 8.50 & - & 7 \\
\hline 21 & $0433+270$ & LP 3 & $\mathrm{D}_{t}$ & 563 & 8.0 & 0.62 & 14. & 9.51 & 18 & 8 \\
\hline 22 & -122 & LP 475-249 & $\mathrm{D}$ & 132 & 8.0 & 0.66 & 11. & 8.50 & 165 & 2 \\
\hline 23 & $0625+415$ & GD 74 & DA & 17610 & 8.07 & 0.66 & 11.10 & $8.12(0.05)$ & 60 & 2 \\
\hline 24 & $0637+477$ & GD 77 & DAP & 14650 (590) & $8.30(0.06)$ & 0.80 & $11.76(0.12)$ & $8.53(0.06)$ & 40 & $2 \mathrm{~B}$ \\
\hline 25 & $0641+438$ & LP $205-27$ & D & 16400 & $8.00(0.05)$ & $0.62(0.03)$ & $11.12(0.08)$ & $8.17(0.05)$ & 76 & 2 \\
\hline 26 & $0743+442$ & GD 89 & DA & $15220(350)$ & $8.47(0.05)$ & $0.91(0.03)$ & $11.98(0.09)$ & $8.61(0.05)$ & 39 & 2 \\
\hline 27 & $0816+376$ & GD 90 & DAP & 11000 (630) & $8.00(0.50)$ & 0.60 & 11.87 & 8.67 & - & 9 \\
\hline 28 & $0233-083.1$ & SDSS J023614.44-080804.9 & $\overline{\mathrm{DQA}}$ & $10000(1000)$ & $8.00(0.50)$ & 0.60 & 12.18 & 8.78 & - & 10 \\
\hline 29 & $0300-083.1$ & SDSS J030325.22-080834.9 & DA & $11550(200)$ & $8.55(0.08)$ & $0.96(0.05)$ & $12.62(0.15)$ & $9.00(0.09)$ & - & 11 \\
\hline 30 & - & LP 652-342 & - & $10940(2840)$ & $8.00(0.50)$ & 0.60 & 11.88 & 8.68 & - & 6 \\
\hline 31 & $0533+322$ & G98-18 & DA & $16970(280)$ & $7.59(0.05)$ & $0.43(0.02)$ & $10.47(0.08)$ & $8.09(0.03)$ & 159 & 2 \\
\hline 32 & $0543+436$ & G96-53 & DAZ & $8260(130)$ & $7.46(0.13)$ & $0.36(0.05)$ & $12.19(0.19)$ & $9.03(0.07)$ & 99 & 2 \\
\hline 33 & 237 & G104-10 & DA & 836 & 8.1 & 0.70 & 11) & $9.10(0.05)$ & 56 & 2 \\
\hline 35 & $58+208$ & SDSS J080114.02+204340.3 & DA & 0) & $0)$ & $1.03(0.12)$ & $14.35(0.38)$ & 9.56 & - & 12 \\
\hline 36 & $0816+387$ & G111-71 & $\mathrm{DA}+\mathrm{dM}$ & 770 & & $0.64(0.04)$ & $13.31(0.12)$ & 9.12 & 45 & $2 \mathrm{~A}$ \\
\hline 37 & $0820+250.1$ & SDSS J082346.14+24 & DA & $34050(100)$ & $7.74(0.02)$ & $0.53(0.01)$ & $9.29(0.03)$ & $6.80(0.01)$ & - & 11 \\
\hline 38 & 54 & PHL 1358 & $\overline{\mathrm{DA}}$ & 70) & 8.58 & 0.98 & $11.94(0.09)$ & $8.52(0.04)$ & 29 & 2 \\
\hline 39 & 01 & G99-37 & DQP & 6130( & 8.18 & $0.69(0.03)$ & $14.42(0.10)$ & $9.56(0.05)$ & 11 & 8 \\
\hline 40 & $0648+368$ & GD 78 & DC & $5700(1000)$ & $8.00(0.50)$ & 0.59 & 14.51 & 9.42 & - & 13 \\
\hline
\end{tabular}

Notes. Star Nos. and categories are the same as in Paper II. Nos. 1 to 10 are the so-called classical Hyades white dwarfs. Nos. 11 to 27 are new candidates (including star Nos. 23 to 27 that are more than 20 pc away from the centre in the $Z$ direction). Nos. 28 to 37 are rated probable non-Hyades members. Nos. 38 to 40 are new candidates identified in Sect. 2 with a residual velocity upper limit of $8 \mathrm{~km} \mathrm{~s}^{-1}$.

References. (1) Sion et al. (2012); (2) Gianninas et al. (2011); (2A) same as previous but with a M-dwarf component removed; (2B) same as previous but magnetic white dwarf fitted with non-magnetic models; (3) Burleigh et al. (1998); (4) Bergeron et al. (2011); (5) photometric fit for $T_{\text {eff }}$, this work, and mass from Silvestri et al. (2001); (6) photometric fit, this work; (7) Koester et al. (2009b); (8) Giammichele et al. (2012); (9) Limoges \& Bergeron (2010); Jordan (1993); (10) Vanlandingham et al. (2005); (11) Tremblay et al. (2011a); (12) Eisenstein et al. (2006); (13) Angel et al. (1981).

\subsection{New selection of Hyades candidates}

The kinematic distances of the Hyades white dwarf candidates were obtained in Paper II by the requirement of the minimum difference between their tangential velocity and the cluster motion, with an upper limit of the residual velocity of $5 \mathrm{~km} \mathrm{~s}^{-1}$. Later in this work (see Sect. 5 below) we recalculate residual velocities of the candidates by assuming the spectroscopic distances derived in Sect. 4. We found that most of the candidates outside of the tidal radius have residual velocities up to $8 \mathrm{~km} \mathrm{~s}^{-1}$. Therefore, we conclude that an upper limit of $5 \mathrm{~km} \mathrm{~s}^{-1}$ for the residual velocity may have been a too rigorous requirement for detecting escaping white dwarfs.

To obtain a complete sample of possible Hyades candidates, we revisited the selection process of Paper II by using a higher upper limit of $8 \mathrm{~km} \mathrm{~s}^{-1}$ for the residual velocity. We found
14 additional white dwarfs for which the kinematic requirement was fulfilled. All but three candidates were later rejected by spectroscopic criteria. The three promising candidates are listed as star Nos. 38 to 40 at the bottom of Table 1 .

\section{Spectroscopic and photometric stellar parameters}

An improved diagnostic of the Hyades membership of white dwarfs can be made based on known stellar parameters. In Table 1, we have compiled the $T_{\text {eff }}, \log g$ and total stellar masses found in the literature for 37 of the 40 candidates. The corresponding absolute $V$ magnitudes $\left(M_{V}\right)$ and white dwarf cooling time $(\log \tau)$ have then been computed using the evolutionary models with thick layers (hydrogen-rich atmosphere DA white 
dwarfs) and thin layers (helium-rich atmosphere DB white dwarfs) of Fontaine et al. (2001, carbon-oxygen cores) for $T_{\text {eff }}<$ $30000 \mathrm{~K}$ and Wood (1995, carbon cores) above this temperature. When available in the cited literature, we also compiled the distances, although we note that the calculation of improved distances is the goal of our Sect. 4 below. Omitted from Table 1 is the binary white dwarf WD $0217+375$ (No. 13), which was designated as an M5V+DA pair without a detailed spectral analysis of the white dwarf (Silvestri et al. 2005). Also omitted is LP 649-0071 (No. 12), a M dwarf star with a suspected but spectroscopically unconfirmed white dwarf companion, previously identified in Luyten's White Dwarf Catalogues (Luyten 1977). Finally, the unconfirmed white dwarf and probable nonmember 1RXSJ062052.2+132436 (No. 34) also has no precise atmospheric parameters.

The majority of the candidates are hydrogen-rich DA white dwarfs. The most successful and precise technique to identify the atmospheric parameters of DA white dwarfs is to fit the observed Balmer line profiles with synthetic profiles predicted from model atmospheres, which is called the spectroscopic technique (Weidemann \& Koester 1980; Schulz \& Wegner 1981; Bergeron et al. 1992). As much as 26 objects in Table 1 rely on the spectroscopic analysis of the Balmer lines to identify their atmospheric parameters (references 2, 7,11 and 12 in the table). Among them, 22 are from the White Dwarf Catalog survey of Gianninas et al. (2011) and two are from the updated SDSS analysis of Tremblay et al. (2011a). Both of these analyses adopted the most recent model atmospheres of Tremblay \& Bergeron (2009), relying on Balmer line profiles including non-ideal gas effects. The quoted uncertainties in Table 1 are from the fitting procedure. However, more realistic estimates of the uncertainties can be made by fitting different observations of the same objects, and Liebert et al. (2005) found spreads of $1.2 \%$ and 0.038 dex in $T_{\text {eff }}$ and $\log g$, respectively. For the remaining of this work, we use these values as the minimum uncertainties on the atmospheric parameters. We note that possible systematic uncertainties from the models are not included. Yet, promising Hyades candidates (Nos. 1-27) with spectroscopic parameters in Table 1 have radiative atmospheres and are outside the range of the socalled high-mass problem (Koester et al. 2009a; Tremblay et al. $2011 \mathrm{~b})$. For these objects $\left(T_{\text {eff }}>13000 \mathrm{~K}\right)$, the atmospheric parameters are in good agreement with the few observational constraints, such as parallax measurements (see, e.g., Fig. 14 of Tremblay \& Bergeron 2009). We emphasize that the current work can also serve as a validation for recent DA models since the distance to the Hyades centre is fairly well constrained via Hipparcos (van Leeuwen 2009). Finally, the helium and hydrogen lines in the DBA star WD $0437+138$ have been fitted spectroscopically by Bergeron et al. (2011) with improved helium-rich models.

Among the remaining candidates, WD $0433+270$ (No. 21) is a cool DA with only a faint $\mathrm{H} \alpha$ line for which a spectroscopic fit is not possible. The BVRIJHK photometric energy distribution of this object has been fitted by Bergeron et al. (2001) to determine $T_{\text {eff }}$ and also the $\log g$ using the constraint from the trigonometric parallax. We use here the results from Giammichele et al. (2012) who refitted the data using models with the Lyman $\alpha \mathrm{H}-\mathrm{H}_{2}$ neutral broadening (Kowalski $\&$ Saumon 2006). The best fit parameters were used to predict a $\mathrm{H} \alpha$ line profile that is in close agreement with the observed one (see Fig. 11f from Giammichele et al.), and therefore we can consider that the atmospheric parameters for this object are similarly precise as those found with the spectroscopic method for the previously discussed stars. The cool and nearby magnetic DQP WD 0548-001 (No. 39) has also been fitted by Giammichele et al. (2012) using a similar method, although with an additional free parameter due to the presence of carbon in the helium-rich atmosphere. All in all, 29 of the 37 objects in Table 1 are typical DA, DB or DQ with well constrained stellar parameters. We remind the reader, however, that two magnetic objects (Nos. 24 and 39) and two objects that have been fitted without the improved Stark broadening profiles (Nos. 20 and 35) likely have underestimated uncertainties.

Other objects in Table 1 have more uncertain atmospheric parameters. WD $0816+376$ (No. 27) is a DAP white dwarf with a high magnetic field that is part of the Kiso survey sample of Limoges \& Bergeron (2010). Even if the object is included in their final sample, they used the estimation of Jordan (1993) for the temperature and fixed the gravity at $\log g=8.0$. Therefore, we chose a characteristic uncertainty of 0.5 dex for the gravity of this object. The situation is similar for the poorly studied cool DC remnant WD 0648+368 (No. 40) and the DQA SDSS J023614.44-080804.9 (probable non-member) for which we also fixed the gravity.

Of the 4 objects in Table 1 that are unresolved binaries (Nos. 1, 4, 7 and 36), WD 0429+176 and WD 0816+387 can be fitted relatively well using the spectroscopic method by removing iteratively the $\mathrm{M}$ dwarf contribution. In contrast, the classical remnants WD $0347+171$ and WD 0418+137 have Fand K-type main-sequence companions that completely dominate the optical flux. As a consequence, these two white dwarfs were observed in the UV to constrain their atmospheric parameters, and the spectroscopic method applied to the Lyman lines is not as well documented and precise as for the Balmer line analysis (Vennes et al. 2005). For one of them, the gravity could not be constrained from the Lyman $\alpha$ spectrum alone, and we chose a characteristic value of $\log g=8.0 \pm 0.5$.

We have at hand, from the PPMXL and Paper II, a photometric data set with Johnson $B V$, CMC14 $r^{\prime}$ and 2MASS $J H K_{\mathrm{S}}$ magnitudes for most objects in Table 1 . We therefore proceeded to fit the photometric data with grids of pure- $\mathrm{H}$ and pure-He white dwarf models, with $T_{\text {eff }}$ and the solid angle $R / D$, where $R$ is the stellar radius and $D$ the distance from the Sun, as free parameters. This method is not sensitive to the gravity, and we used a fixed value of $\log g=8.0$. Consequently, this method can only be used to give an independent value ${ }^{2}$ of $T_{\text {eff }}$, called photometric temperature below. We rely on the prescription of Holberg \& Bergeron (2006) to convert the magnitudes into average fluxes.

From our photometric fits, we find that in all but one case the photometric temperature agrees with the spectroscopic value. The exception is WD 0231-054 for which the JHK flux appears too high and suggests a lower $T_{\text {eff }}$ for this object. We have no clear explanation for this behaviour. Most objects in Table 1 are hot and blue, and their colors, in the Rayleigh-Jeans regime, are not very sensitive to $T_{\text {eff }}$ (or the absolute magnitude, as can be seen in Fig. 1). Therefore, the photometric temperatures are less precise and we rely, whenever possible, on spectroscopically derived temperatures. There are four cases where no spectroscopic parameters are available. The first is the DA WD 0120-024 (No. 11), which has only been fitted using the $V-I$ color in Silvestri et al. (2001). On the contrary, our data set includes 2MASS $J H K_{\mathrm{S}}$ photometry, and we could also find for this object the Johnson BVRI photometry (Smith 1997). Our fit is shown in Fig. 2 with the gravity fixed at the value found by Silvestri et al. (2001) from a gravitational redshift measurement. We note

2 Note that the distances could also be determined with this method, but the technique described in Sect. 4 is more precise. 
P.-E. Tremblay et al.: Spectroscopic and photometric studies of white dwarfs in the Hyades

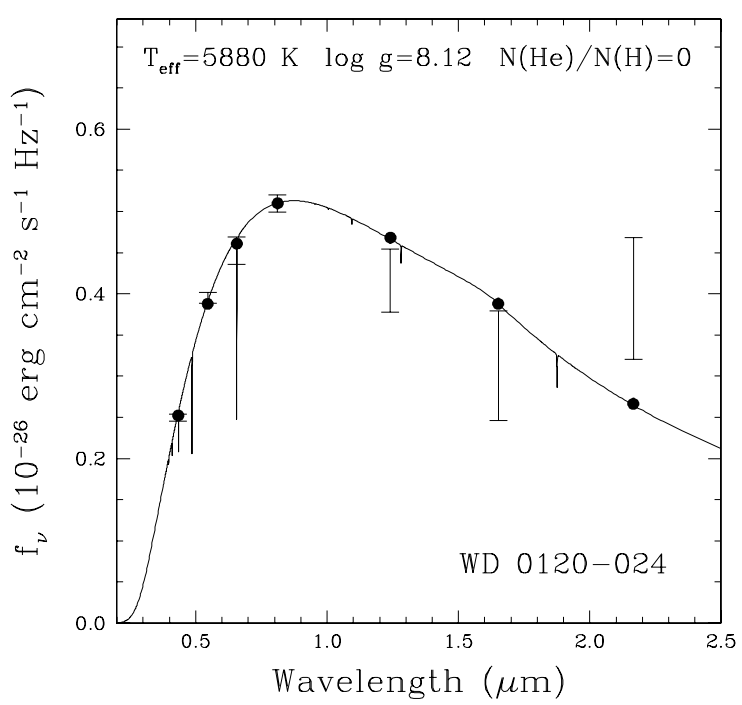

Fig. 2. Johnson $B V R I$ and $2 \mathrm{MASS} J H K_{\mathrm{S}}$ photometry of WD 0120-024 (error bars) fitted by average model fluxes (filled points) for a purehydrogen atmosphere. The best fit parameters are given in the figure, and the corresponding predicted spectrum is also shown (solid line).
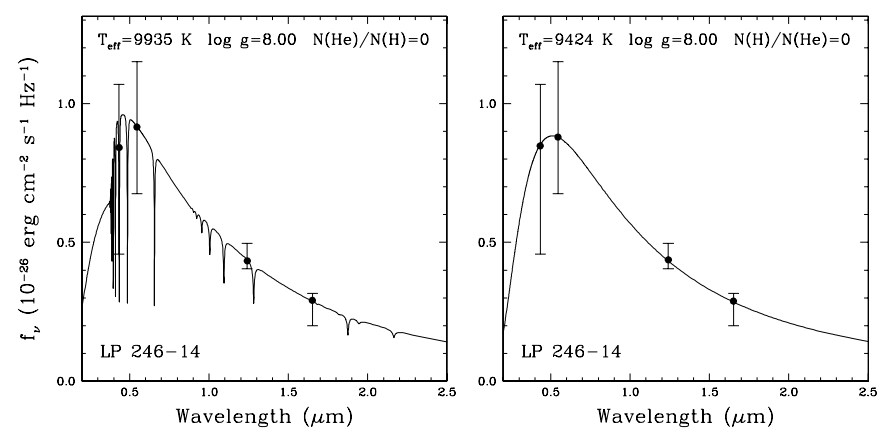

Fig. 3. Similar to Fig. 2 but for the $B V$ and 2MASS $J H$ fit of LP 246-14 using pure-hydrogen models (left panel) and pure-helium models (right panel).

a discrepancy at the $K_{\mathrm{S}}$ band, which is not unusual as it is close to the detection limit of 2MASS. The atmospheric parameters should be considered as relatively well constrained for this object, despite the fact that masses determined from gravitational redshifts are generally not as precise as those found from spectroscopic or parallax measurements (Bergeron et al. 2007).

In Fig. 3 we show our $B V$ and 2MASS $J H$ fit of LP 246-14 (No. 15), which is found in the Luyten's White Dwarf Catalogues but is not a spectroscopically confirmed white dwarf, and no atmospheric parameters have yet been published. The fit is consistent with a relatively cool white dwarf, but the lack of a spectrum for this object prevents us to conclude that it is a white dwarf. While in the next section it is shown that the $d_{\text {kin }}$ value is consistent with a gravity of $\log g \sim 8$, it does not exclude that it is a background lower gravity object with a high tangential velocity. Therefore, the atmospheric parameters presented in Table 1, an average of the pure-H and pure-He solutions, should be used with high caution until a spectrum is secured. Among the other possible but unconfirmed white dwarfs, we derived the atmospheric parameters of LP 652-342 (probable non-member) in a similar fashion. For 1RXSJ062052.2+132436, we find that the photometry is consistent with a very hot object, but it is in a regime where $T_{\text {eff }}$ can not be estimated with a reasonable accuracy, and therefore it is omitted from Table 1.

\section{Spectroscopic and kinematic distances}

The set of atmospheric parameters that we have compiled in Table 1 can be used, along with model atmospheres, to predict absolute magnitudes for any photometric band. By combining these values with observed magnitudes, we can compute the socalled spectroscopic distances $\left(d_{\text {spectro }}\right)^{3}$. This is independent of the kinematic distances derived in Paper II to which they can be compared. Our photometric data set, including 2MASS $J H K_{\mathrm{S}}$, CMC14 $r^{\prime}$ and Johnson $B V$ can be used to predict as much as 6 alternative $d_{\text {spectro }}$ values. In Fig. 4 we present, for all observed magnitudes, the ratio of the spectroscopic and kinematic distances for objects in Table 1. In the computation of the uncertainties, we account for the contribution of the $d_{\text {kin }}$ prediction (depending only on the observed proper motion and sky position), the atmospheric parameters and the observed magnitude. We note that unresolved binaries are omitted from Fig. 4, since the observed magnitudes are all either mildly or strongly contaminated by the companion.

It is clear that the different $d_{\text {spectro }}$ determinations for a same object are not completely independent, since they are based on the same set of atmospheric parameters. Nevertheless, by looking at the results between different filters, one can get an overview of the quality of the photometric data sets. It is seen that the scatter is in general low between the different filters, implying that our photometric data sets are fairly homogeneous and well calibrated. We nevertheless note that one $K_{\mathrm{S}}$ magnitude (No. 16) and one $H$ magnitude (No. 20) in Fig. 4 are significantly different to other values. Tremblay \& Bergeron (2007) have shown that one must be careful with spurious magnitude values in 2 MASS close to the survey detection limit, and these are unlikely to be the signature of real near-infrared spectral features. Furthermore, $B$ and $V$ magnitudes are drawn from different sources with inhomogeneous accuracy, and often even have no quoted uncertainties ${ }^{4}$. Based on these facts, we decided to

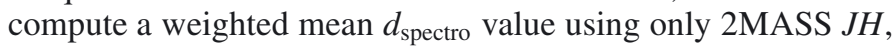
CMC $r^{\prime}$ and $V$ magnitudes (when the uncertainties are given) ${ }^{5}$. In Fig. 4 , we show the comparison between the mean $d_{\text {spectro }}$ and $d_{\text {kin }}$ determinations for each candidate. These quantities are also given in Table 2, along with the number of filters involved in the $d_{\text {spectro }}$ mean. Note that at this point, we leave out from our calculations and discussion the objects labelled as probable non-members in Paper II (Nos. 28-37), since they are clearly all background objects according to Fig. 4.

In the next section we will discuss about the Hyades membership of new candidates based on the results of Fig. 4. Beforehand, there is some interesting information that can be extracted from the classical members. The non-binary Hyades white dwarfs (Nos. 2-3, 5-6 and 8-10) can serve as a benchmark to evaluate the precision of our analysis. The spread due to the photometric uncertainties has already been evaluated from Fig. 4. It is now seen from the same figure that the mean $d_{\text {spectro }}$ values derived in this work are in good agreement, roughly within the uncertainties, with $d_{\text {kin }}$ values. The spread around the expected unity ratio can be used to learn

\footnotetext{
${ }^{3}$ Note that in a few cases, photometric instead of spectroscopic fits were used to determine the atmospheric parameters, but we use the term spectroscopic distance in this work for clarity.

${ }^{4}$ Our results indicate that $B$ and $V$ magnitudes are nevertheless in fairly good agreement with the other data sets, and that the often used typical average uncertainty of $0.05 \mathrm{mag}$ in $V$ (see, e.g., Tremblay \& Bergeron 2008) appears adequate.

${ }^{5}$ For stars No. 22 and 40, we assume a $V$ uncertainty of 0.2 since no 2MASS or CMC measurements are available.
} 


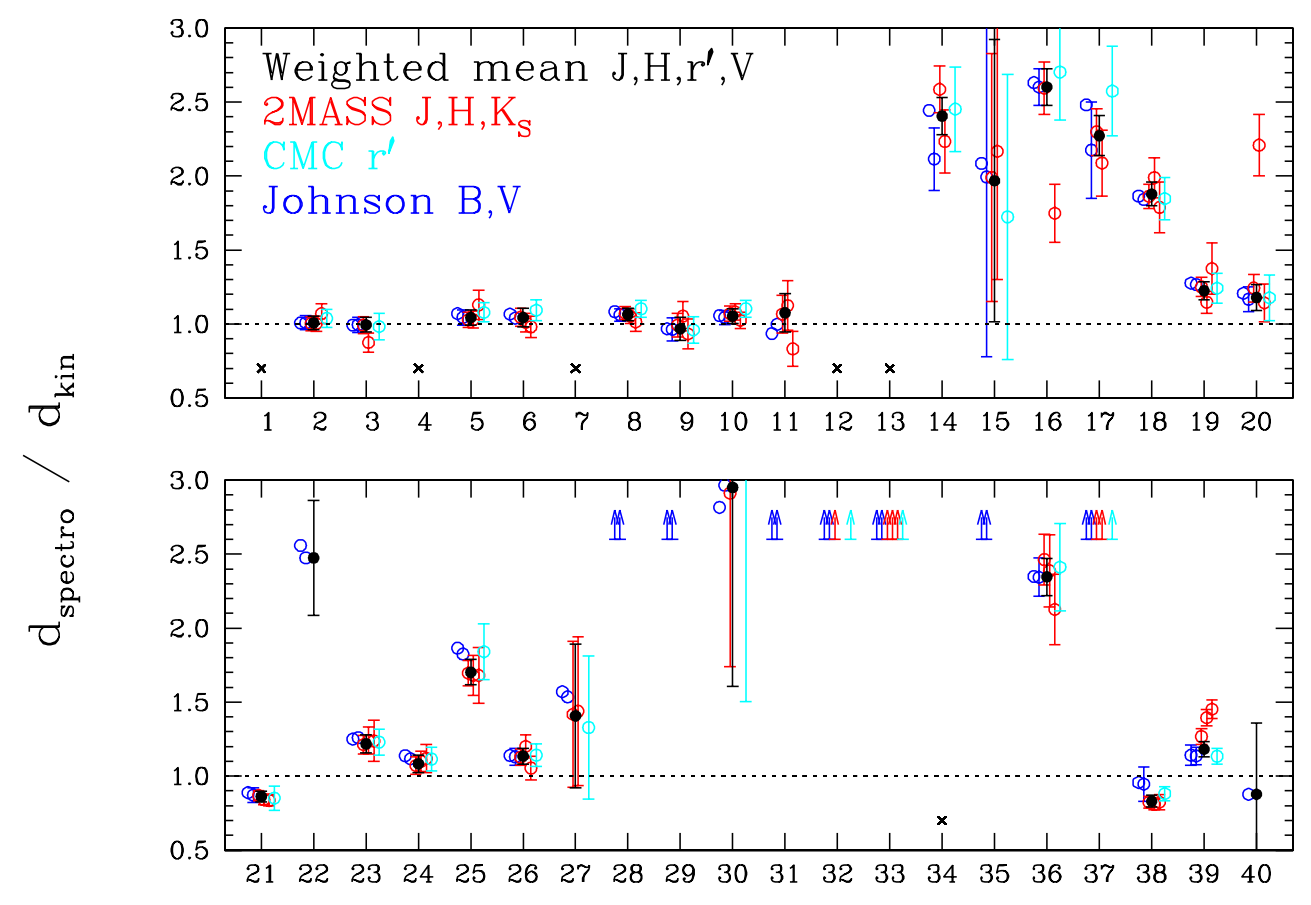

\section{Star number}

Fig. 4. Ratio of the spectroscopic to kinematic distance for the Hyades white dwarf candidates identified by their star number from Table 1

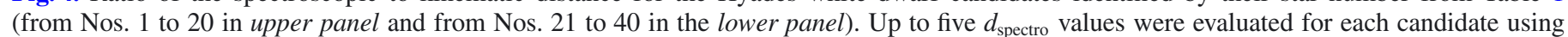
2MASS $J H K_{\mathrm{S}}$ (red), CMC $r^{\prime}$ (cyan) and Johnson $B V$ (blue) photometry (open points). Many $B$ and $V$ magnitudes have no published uncertainties. Also shown is the the mean weighted spectroscopic distance from 2MASS $J H$, CMC $r^{\prime}$ and $V$ filters (black filled points). The data points are shifted horizontally for clarity. Objects with a cross above the star No. are binaries (Nos. 1, 4, 7, 12 and 13) or have no atmospheric parameters (No. 34). For several objects, the ratio is actually higher than the scale of the plot, and is represented by a lower limit symbol. The dotted horizontal line represents a perfect match between both distance estimates.

about the combined uncertainties due to spectroscopic parameter determinations and $d_{\text {kin }}$ predictions. The good agreement suggests that the uncertainties in this work are relatively well estimated and that our method should be fairly robust to argue on the status of the Hyades membership of new candidates. Furthermore, we note that the single classical members have a mean mass of $0.75 M_{\odot}$, significantly higher than the value of $0.638 M_{\odot}$, with a dispersion of $0.145 M_{\odot}$, found for field white dwarfs (Gianninas et al. 2011). Also, their cooling ages are in the range 10-340 Myr, which is consistent with the young age of the Hyades, though significantly smaller than the estimated age of the cluster of 625 Myr. According to Dobbie et al. (2006), this corresponds to main-sequence progenitors in the range of $\sim 2.5-4 M_{\odot}$. Therefore, more massive and slightly older white dwarfs, hence fainter and cooler objects, could be expected among the new candidates.

One uncertainty that is often difficult to evaluate, and not included in Fig. 4, is the precision of the model atmospheres. The classical Hyades white dwarfs, however, can help us for the evaluation of this uncertainty. Since any possible inaccuracies in the model atmospheres are expected to cause a systematic offset of the atmospheric parameters, they would also cause systematic shifts of the points in Fig. 4. Other uncertainties discussed so far are much more likely to cause a spread around a mean value. Our results show that there is no need to account for an additional offset, and therefore we conclude that the atmospheric parameters are sufficiently accurate on the average. This is a fairly precise validity verification of the physics in the Tremblay \& Bergeron (2009) DA models, and to a lesser degree the Bergeron et al. (2011) DB models (since only star No. 9 is helium-rich), which were adopted to predict the atmospheric parameters of classical members. Reversing this statement, it illustrates that the method of using model atmospheres and spectroscopic observations to

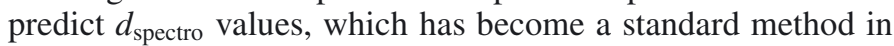
recent years (see, e.g., Holberg et al. 2008; Dobbie et al. 2009; Williams et al. 2009), is fairly well calibrated and precise on average. One still has to be cautious about the precision of observed magnitudes used in this process.

\section{Membership status of new Hyades candidates}

We have tested our $d_{\text {spectro determinations with the classical }}$ Hyades, and we expect these values to be equally precise for the new aspirants. In this section, we review the status of the 17 new candidates (Nos. 11-27) identified in Paper II and the 3 new candidates (Nos. 38-40) identified in this work. For that purpose, we have computed in Table 2 the new distances between the objects and the Hyades centre ( $\left.d_{\text {Hyades centre }}\right)$ based on the $d_{\text {spectro }}$ values. It is clear that all but one (No. 20) of the new candidates are outside of the tidal radius.

Another quantity in Table 2 derived from the $d_{\text {spectro }}$ calculation is the residual tangential velocity $\left|v_{\text {tang }}\right|$ with respect to the expected motion of the star towards the cluster convergent point. In Fig. 5, the residual velocity of the candidates is plotted versus the distance to the cluster centre. Our updated $\left|v_{\text {tang }}\right|$ values are by definition higher than those found in Paper II, where the difference in the proper motion of candidates and that of the Hyades where minimised on a plane perpendicular to the line-of-sight, to derive the kinematic distances. Also included in Fig. 5 are the two unresolved binaries for which we have no atmospheric 
Table 2. Distances of Hyades white dwarf candidates.

\begin{tabular}{|c|c|c|c|c|c|c|c|}
\hline Star No. & Name & $\begin{array}{l}d_{\text {kin }} \\
(\mathrm{pc})\end{array}$ & $\begin{array}{c}d_{\text {spectro }} \\
(\mathrm{pc})\end{array}$ & Filters $^{a}$ & $\begin{array}{l}\left|v_{\text {tang }}\right|^{b} \\
\left(\mathrm{~km} \mathrm{~s}^{-1}\right)\end{array}$ & $\begin{array}{c}d_{\text {Hyades centre }}^{b} \\
\text { (pc) }\end{array}$ & Status $^{c}$ \\
\hline 2 & WD 0352+096 & $35.1(0.7)$ & $35.4(1.4)$ & 4 & $1.0(0.9,1.7)$ & $13.3(12.2,14.4)$ & classical \\
\hline 3 & WD 0406+169 & $51.8(1.6)$ & $51.5(2.0)$ & 4 & $0.5(0.4,1.2)$ & $6.5(5.0,8.2)$ & classical \\
\hline 5 & WD $0421+162$ & $44.1(1.4)$ & $46.0(1.7)$ & 4 & $1.4(0.9,2.2)$ & $1.0(0.9,2.2)$ & classical \\
\hline 6 & WD $0425+168$ & $47.8(2.1)$ & $49.9(2.0)$ & 3 & $1.2(0.6,2.2)$ & $3.6(1.6,5.6)$ & classical \\
\hline 8 & WD $0431+126$ & $45.7(0.4)$ & $48.6(1.9)$ & 4 & $1.5(0.6,2.4)$ & $4.2(3.4,5.5)$ & classical \\
\hline 9 & WD 0437+138 & $46.9(1.8)$ & $45.4(3.2)$ & 4 & $1.0(0.7,2.2)$ & $3.3(3.2,5.2)$ & classical \\
\hline 10 & WD $0438+108$ & $46.1(1.1)$ & $48.6(1.9)$ & 4 & $1.2(0.4,2.0)$ & $5.8(5.3,6.9)$ & classical \\
\hline 11 & WD 0120-024 & $39.1(0.8)$ & $42.0(5.0)$ & 2 & $3.5(0.8,9.1)$ & $37.1(35.9,39.0)$ & old WD \\
\hline 14 & WD $0230+343$ & $37.9(0.6)$ & $91.1(4.5)$ & 4 & $56.0(51.3,60.8)$ & $56.8(52.8,60.9)$ & non-member \\
\hline 15 & LP 246-14 & $35.1(0.5)$ & $69.1(33.4)$ & 4 & $37.9(2.5,75.0)$ & $36.8(23.3,66.3)$ & old WD \\
\hline 16 & WD 0259+378 & $66.2(1.8)$ & $172.3(6.9)$ & 3 & $61.9(57.9,65.8)$ & $133.4(126.6,140.2)$ & non-member \\
\hline 17 & WD 0312+220 & $44.4(1.0)$ & $101.0(5.6)$ & 4 & $45.3(40.9,49.7)$ & $58.7(53.3,64.1)$ & non-member \\
\hline 18 & WD 0339-035 & $28.5(0.4)$ & $53.5(2.1)$ & 3 & $27.5(25.4,29.8)$ & $21.2(20.2,22.4)$ & non-member \\
\hline 19 & WD 0348+339 & $38.5(0.7)$ & $47.1(2.1)$ & 3 & $7.3(5.6,9.1)$ & $15.6(15.3,16.2)$ & candidate \\
\hline 20 & HS $0400+1451$ & $38.5(2.5)$ & $45.2(1.3)$ & 3 & $5.1(4.2,6.0)$ & $5.1(5.0,5.4)$ & candidate \\
\hline 21 & WD 0433+270 & $20.1(0.2)$ & $17.3(0.7)$ & 4 & $3.6(2.7,4.5)$ & $29.5(28.8,30.2)$ & old WD \\
\hline 22 & WD 0437+122 & $67.1(7.7)$ & $166.1(17.9)$ & 1 & $31.7(26.0,37.4)$ & $120.0(102.2,137.9)$ & non-member \\
\hline 23 & WD $0625+415$ & $47.6(1.4)$ & $58.0(2.4)$ & 3 & $6.6(5.5,7.9)$ & $34.0(32.6,35.5)$ & candidate \\
\hline 24 & WD 0637+477 & $36.2(0.7)$ & $39.3(2.1)$ & 3 & $3.0(1.7,4.6)$ & $30.9(30.6,31.2)$ & candidate \\
\hline 25 & WD $0641+438$ & $41.8(1.1)$ & $71.2(3.1)$ & 3 & $19.9(17.9,21.9)$ & $46.2(43.8,48.6)$ & non-member \\
\hline 26 & WD $0743+442$ & $33.6(0.6)$ & $38.1(1.7)$ & 4 & $4.3(2.9,5.8)$ & $36.4(36.0,36.8)$ & candidate \\
\hline 27 & WD 0816+376 & $38.8(0.9)$ & $54.5(18.8)$ & 3 & $11.8(0.9,25.9)$ & $46.8(38.8,59.9)$ & uncertain \\
\hline 38 & WD 0231-054 & $32.8(0.4)$ & $27.2(1.3)$ & 3 & $8.6(7.6,9.7)$ & $29.0(28.6,29.5)$ & candidate \\
\hline 39 & WD 0548-001 & $9.4(0.2)$ & $11.1(0.4)$ & 3 & $6.8(6.4,7.1)$ & $36.7(36.4,37.1)$ & old WD \\
\hline 40 & WD 0648+368 & $36.2(1.0)$ & $31.8(17.4)$ & 1 & $5.7(5.6,14.1)$ & $28.6(28.2,36.0)$ & old WD \\
\hline
\end{tabular}

Notes. ${ }^{(a)}$ Number of photometric measurements used to compute the mean spectroscopic distance. ${ }^{(b)}$ Numbers in parentheses are not rms errors but refer to the minimum and maximum values where the distance of a given white dwarf is varying within $d_{\text {spectro }} \pm 1 \sigma .{ }^{(c)}$ Summary of the status of the Hyades membership as discussed in this paper, including the classical members and the new candidates that are still valid candidates or that were rejected as non-members following our analysis. We also identify old white dwarfs for which the age is incompatible with a Hyades origin. Finally, one object has uncertain atmospheric parameters and we can not rule it out as a member. Note that binaries (three classical members, Nos. 1, 4 and 7, and two new candidates, Nos. 12 and 13) are excluded from this table.

parameters (Nos. 12 and 13), and we use their kinematic parameters to have the complete picture of possible members. Because the companion of these candidates follow very well the Hyades main sequence in the absolute magnitude vs. color diagram for near-infrared colors (see Fig. 4 of Paper I), their kinematic distances are expected to be fairly accurate.

According to Fig. 4 , the $d_{\text {spectro }}$ and $d_{\text {kin }}$ determinations do not agree within $1 \sigma$ for most candidates, which would imply that their membership is doubtful. On the other hand, if we allow them to have residual velocities of a few kilometers per second, they could still be valid unbound constituents. As a consequence, the residual velocities of Fig. 5 are better suited to look at the membership status of new candidates. The residual velocities are of the order of $\sim 1 \mathrm{~km} \mathrm{~s}^{-1}$ for the classical members, and one could in principle use this characteristic value for a membership confirmation. However, Paper I has shown that unbound main-sequence stars had a significantly higher residual velocity dispersion than the bound members, and we can expect a similar behaviour for white dwarfs. Then, a higher residual velocity can be allowed to judge the status of the new candidates.

It is already clear that 6 of the candidates (star Nos. 14, 16, 17, 18, 22 and 25) must be ruled out as Hyades components, since their updated distances imply residual velocities of more than $20 \mathrm{~km} \mathrm{~s}^{-1}$. Indeed, it is obvious that a cluster candidate can not have a residual velocity of the order of the velocity dispersion of field white dwarfs, which is $\sim 30 \mathrm{~km} \mathrm{~s}^{-1}$ in each spatial direction (Sion et al. 2009).

Before we look at the remaining candidates, it is desirable to understand why the above 6 background objects contaminated

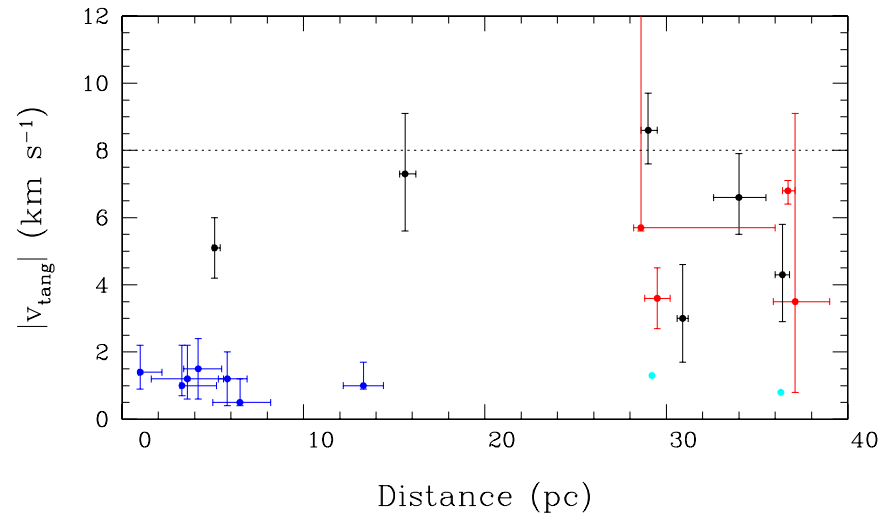

Fig. 5. Residual tangential velocity $\left|v_{\text {tang }}\right|$ with respect to the Hyades group motion as a function of the distance to the cluster centre, for classical members (blue points and error bars) and new candidates with an age lower (black points and error bars) and higher (red points and error bars) than the Hyades age. Also shown are the two candidates in binaries (Nos. 12 and 13, cyan points in the figure) for which we have no spectroscopic parameters. Instead we rely on the kinematic values, providing lower limits on the residual velocities. The error bars are not rms errors but refer to the minimum and maximum values where the distance of a given white dwarf is varying within $d_{\text {spectro }} \pm 1 \sigma$. The horizontal dotted line represents the $8 \mathrm{~km} \mathrm{~s}^{-1}$ residual velocity upper limit for the candidate selection.

the sample of Paper II. From the kinematic selection alone, it is clear that background, high velocity objects, could pollute 


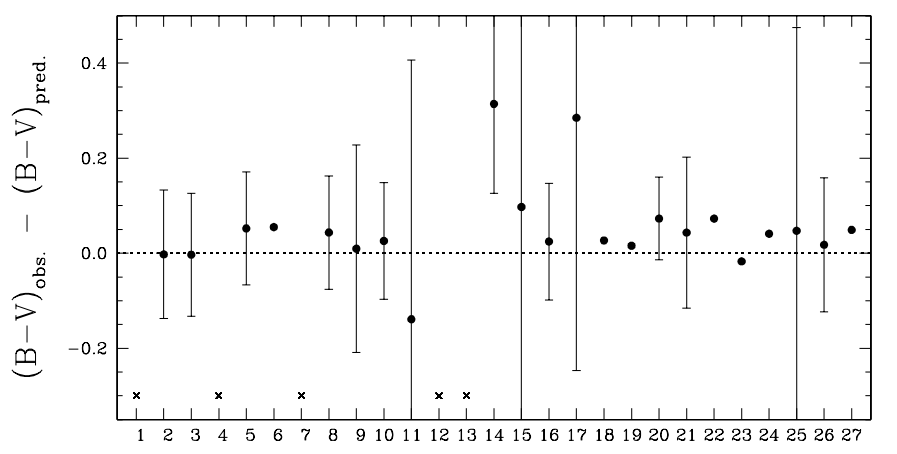

Star number

Fig. 6. Difference in the observed and predicted $B-V$ colors for the Hyades white dwarf candidates identified by their star number. The dotted horizontal line represents a perfect match between both quantities. Objects with a cross above the star No. are binaries.

the sample. However, these background stars should have been removed in the photometric selection, where more distant objects appear subluminous compared to true Hyades members (see Fig. 1). In Fig. 6, we compare the observed and predicted $B-V$ colors, the latter computed from model spectra with the atmospheric parameters from Table 1 . We conclude that only two objects (Nos. 14 and 17) have been misidentified as candidates since their published $B-V$ colors erroneously put them on the white dwarf sequences. For the other four candidates rejected as members, we conclude that they just happened to be within the theoretical sequences by chance, but with a vertical offset. Indeed, for hot white dwarfs, the absolute $V$ magnitude is rather insensitive to the $B-V$ color as can been seen from Fig. 1. More precisely, for a given color, a range of $M_{V}$ of as much as two magnitudes is possible. It implies that it is very difficult, from an absolute magnitude vs. color diagram alone, to conclude if a kinematic candidate is a Hyades member, or a hotter and more distant background white dwarf. The method of comparing kinematic and spectroscopic predictions used in this work is then necessary to obtain a clean sample. One might be intrigued by the fact that we find 6 background objects (up to $172 \mathrm{pc}$ from the Sun), and no foreground objects. The main reason seems that the volume surveyed in this work in front of the cluster is only $\sim 1 / 50$ of the volume beyond the cluster up to the most distant background object. This volume effect may not be important since catalogues of white dwarfs are far from complete at distances greater than the Hyades centre. On the other hand, all of our background objects are hot DAs, and these objects are fairly rare up to a radius of $20 \mathrm{pc}$ (Giammichele et al. 2012) and are more probably found at larger distances.

There remain 14 objects, including 2 binaries with no spectroscopic parameters, that are still possible members. Five candidates (Nos. 11, 15, 21, 39 and 40), however, are significantly older than the age of the cluster, including one object (No. 15) that is not spectroscopically observed and hence confirmed as a white dwarf. Furthermore, we have one magnetic object with imprecise atmospheric parameters (No. 27). According to Fig. 5, most of the remaining candidates have significant residual velocities of the order of $5 \mathrm{~km} \mathrm{~s}^{-1}$ and lie far away outside of the tidal radius. These candidates, if they are of Hyades origin, are not clearly linked to the classical members in terms of their cluster centre distances and residual velocities, but could be escaped former members.

In principle, the diagram of Fig. 5 should be fairly complete up to $8 \mathrm{~km} \mathrm{~s}^{-1}$, although it must be kept in mind that not all white dwarfs around the Hyades have been identified in white dwarf catalogues. The goals of the next sections will be to understand the contamination from field white dwarfs and the completeness of the white dwarf catalogues.

\subsection{Field white dwarfs contamination}

We made Monte Carlo simulations to estimate the number of field white dwarfs that could lie within a radius of $40 \mathrm{pc}$ of the cluster and mimic, by chance, the Hyades motion, hence creating false positive detections. As input, we need the luminosity function of white dwarfs within $\sim 100 \mathrm{pc}$ of the Sun. Such a function is not known, but, based on improved atmospheric parameters of degenerate stars, Giammichele et al. (2012) published the luminosity function of the relatively complete sample of white dwarfs within a $20 \mathrm{pc}$ radius from the Sun. In principle, it would be appropriate to extrapolate their results to the Hyades region. However, the hot end of the luminosity function, which is of significant interest for the young cluster, suffers from low number statistics in the local sample of white dwarfs. Consequently, we rely on the SDSS luminosity function (Harris et al. 2006), which includes a much larger number of hot objects, but is incomplete by as much as $50 \%$ and complex completeness corrections must be accounted for. Nevertheless, Fig. 22 of Giammichele et al. (2012) shows that the local sample and SDSS luminosity functions agree relatively well, and in particular, the derived total space densities are very similar. Therefore, we simulated white dwarf populations weighted by the SDSS luminosity function in a sphere of $40 \mathrm{pc}$ radius centred on the Hyades. The objects are randomly distributed in the galactic $X, Y, Z$ coordinates, with axes pointing to the Galactic centre $(X)$, the direction of galactic rotation $(Y)$, and the North Galactic Pole $(Z)$. The $U, V, W$ velocities are distributed according to a Gaussian weight function using the observed velocity distribution from Sion et al. (2009) for the local $20 \mathrm{pc}$ sample. They find mean velocities, with respect to the Sun, of $[\langle U\rangle,\langle V\rangle,\langle W\rangle]=[-0.9,-21.5,-5.2] \mathrm{km} \mathrm{s}^{-1}$ and dispersions of $\left[\sigma_{U}, \sigma_{V}, \sigma_{W}\right]=[35.3,33.1,28.1] \mathrm{km} \mathrm{s}^{-1}$. To derive the mean velocity and the velocity dispersion, Sion et al. (2009) assumed zero radial velocity, which, at least, sets a lower limit to the velocity dispersion.

Our 100 simulations yield on average 1233 white dwarfs within $40 \mathrm{pc}$ of the cluster centre. Nonetheless, we do not expect that many of these objects will have velocities close to the Hyades group motion of $[-41.1,-19.2,-1.4] \mathrm{km} \mathrm{s}^{-1}$ (Paper I). On average, we find that 2.6 objects have $3 \mathrm{D}$ velocities that are consistent with the Hyades motion within $8 \mathrm{~km} \mathrm{~s}^{-1}$. However, $3 \mathrm{D}$ velocities can not be measured in most cases due to the lack of radial velocities. Hence we also measured the residual $2 \mathrm{D}$ velocities of our simulated objects with respect to the Hyades motion perpendicular to the line-of-sight. In that case we find on average 29 objects that mimic the cluster tangential motion within $8 \mathrm{~km} \mathrm{~s}^{-1}$. We show one of our simulations in the upper panel of Fig. 7, with the relative $2 \mathrm{D}$ velocity as a function of the cluster centre distance. The lack of objects close to the centre is simply a volume effect. We note that cool white dwarfs dominate the luminosity function and also the local white dwarf sample, and consequently $88 \%$ of our simulated candidates are cool objects $\left(M_{V}>12\right.$, red points on the figure), for which the age, above $\sim 500 \mathrm{Myr}$, would be inconsistent with a Hyades membership. In other words, only about 3 of the contaminants would have an age consistent with the Hyades age. The summary of the predicted number of polluting objects is given in Table 3. Finally, it should be realized that due to the uncertainties in the observed luminosity functions and velocity distributions, 
P.-E. Tremblay et al.: Spectroscopic and photometric studies of white dwarfs in the Hyades

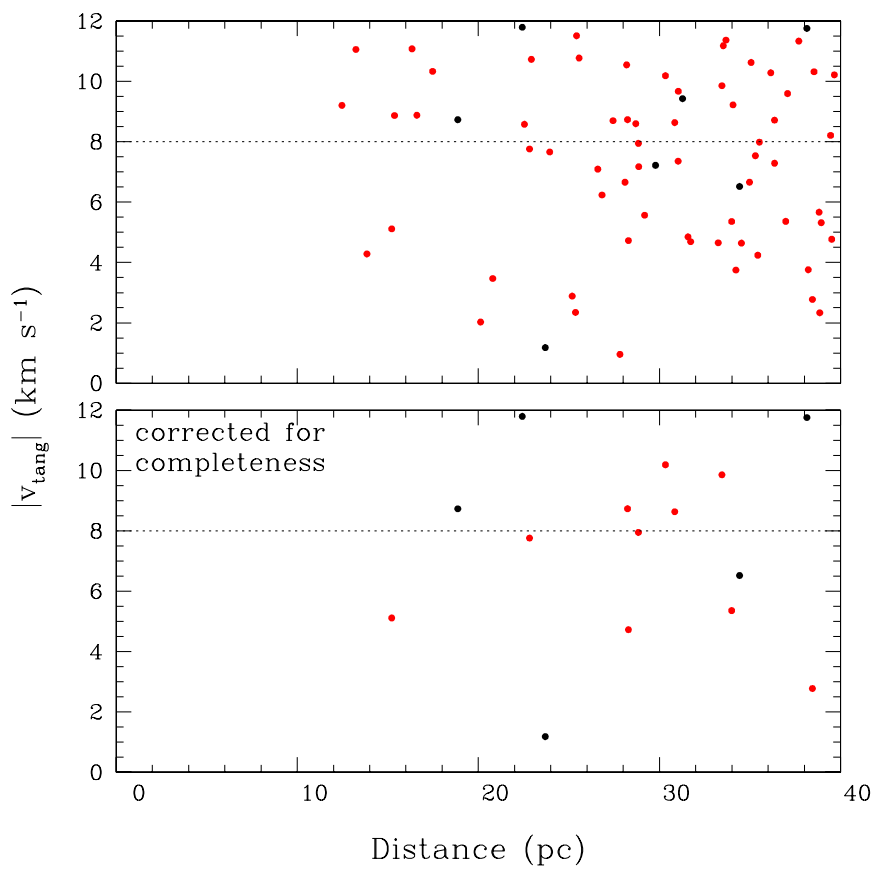

Fig. 7. Top: residual tangential velocity with respect to the Hyades group motion, as a function of the distance to the cluster centre, for a selected Monte Carlo simulation of field white dwarfs as described in the text. The red points are cool white dwarfs with $M_{V}>12$. The horizontal dotted line represents the residual velocity upper limit of $8 \mathrm{~km} \mathrm{~s}^{-1}$ used in Sect. 5.1. Bottom: same simulation as the top panel, but corrected for the completeness of the WDC (see Sect. 5.3).

Table 3. Contamination from field white dwarfs.

\begin{tabular}{lccc}
\hline \hline Residual velocity $\left(<8 \mathrm{~km} \mathrm{~s}^{-1}\right)$ & Total & $M_{V}<12$ & $M_{V}>12$ \\
\hline uncorrected for completeness & & & \\
\hline 3D & 2.6 & 0.30 & 2.3 \\
2D & 29 & 3.3 & 26 \\
\hline corrected for completeness & & & \\
\hline 3D & 0.34 & 0.21 & 0.13 \\
2D & 3.9 & 2.4 & 1.5 \\
\hline
\end{tabular}

Notes. Given a maximum cluster centre distance of $40 \mathrm{pc}$.

the numbers given in this section are only accurate to about the $10 \%$ level.

The selection criteria of candidates in Paper II include a cross-match with the White Dwarf Calatog (WDC) as well as with a few other sources. These catalogues suffer from incompleteness and we have to take this into account in our Monte Carlo simulations. In the following, we therefore investigate the incompleteness of the WDC to determine a realistic estimate of the field star contamination in our sample.

\subsection{Completeness corrections}

The $V$ magnitude distribution of the simulated white dwarfs from the previous section is shown in Fig. 8 (top panel, bold histogram). Since the local sample of white dwarfs is only complete up to about $20 \mathrm{pc}$ and that the objects in our simulations are on average twice as distant, it is already obvious that many of them may be missing in white dwarf catalogues. One important thing to notice, however, is that all of the faint objects with $V>17$ in our simulations are cool white dwarfs with $M_{V}>12$. Indeed, for white dwarfs with $M_{V}<12$, i.e. those with an age

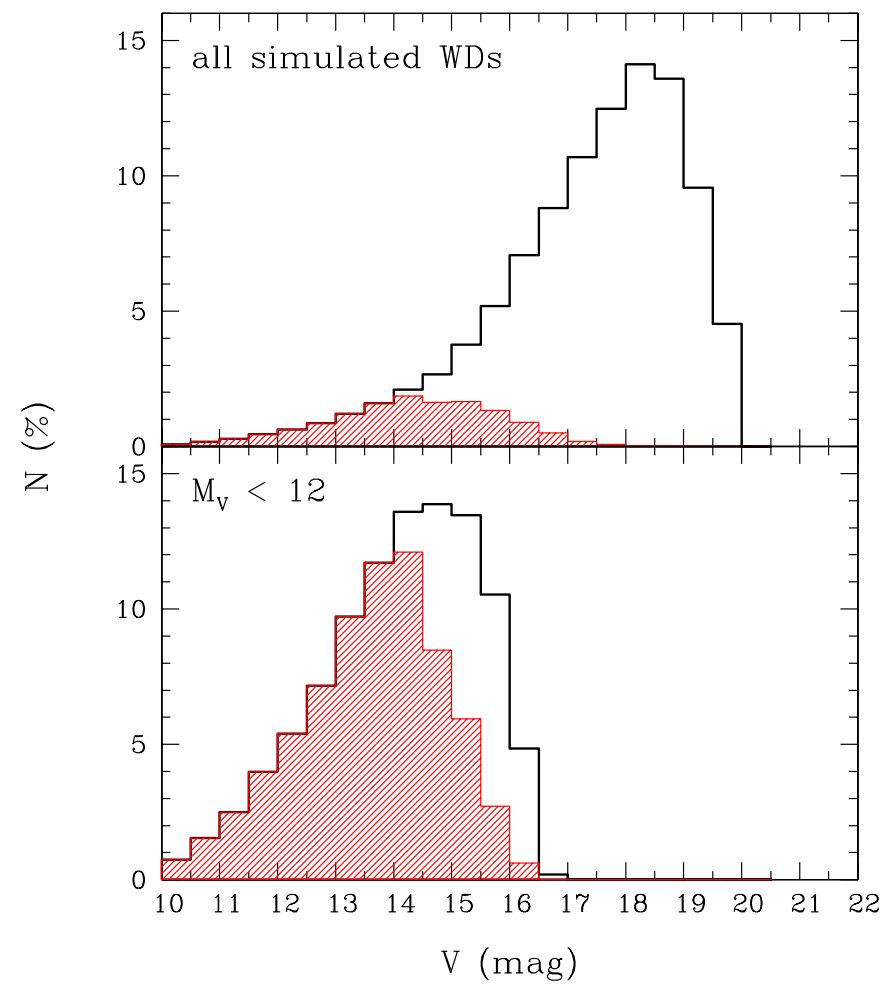

Fig. 8. Top: Distribution of $V$ magnitudes for the simulations of field white dwarfs discussed in Sect. 5.2 (bold histogram). The distribution has then been folded with our estimated completeness fraction of the WDC (red hatched histogram). Bottom: same as top panel but only for objects with $M_{V}<12$.

roughly consistent with the age of the Hyades, the bottom panel of Fig. 8 shows that they are always relatively bright $(V<17)$.

To estimate the completeness of the White Dwarf Catalog, we used the following procedure. We computed the $V$ magnitude histogram for all objects in the catalog 6 , which is presented in Fig. 9 in terms of the logarithm of the number. We neglected all objects first discovered in the SDSS. Removing these objects seems justified to estimate the contamination of the Hyades sample, since all candidates in Table 2 were known before the SDSS era, and SDSS only partly covers the Hyades area. For uniform stellar density, a linear relation, with a slope of 0.6 , is expected if a sample is volume complete. We can see that the observed distribution in the range $12.5<V<14.5$ is fitted well by the expected relation. We computed the completeness at $V>14.5$ from the ratio of observed to predicted objects. Afterwards, we folded this completeness function with the distribution of $V$ magnitudes in our field white dwarfs simulations presented in Fig. 10 (red dashed histograms). We derive from the new total number of objects that only $14 \%$ of the field white dwarfs within a radius of $40 \mathrm{pc}$ to the Hyades centre are found in the WDC. This fraction increases to $72 \%$ if we only account for young white dwarfs with $M_{V}<12$, which implies that the completeness for old stars above this threshold is only about $6 \%$. To find the number of field white dwarfs mimicking Hyades members, we simply have to multiply the completeness fractions to the numbers of the previous section. The result is given in Table 3, and, as an example, in Fig. 7 (bottom panel) where we modified the simulation by allowing objects to randomly disappear in accordance with the completeness correction

${ }^{6}$ We have neglected magnitudes taken prior to 1972, photographic magnitudes, and those flagged as uncertain. 


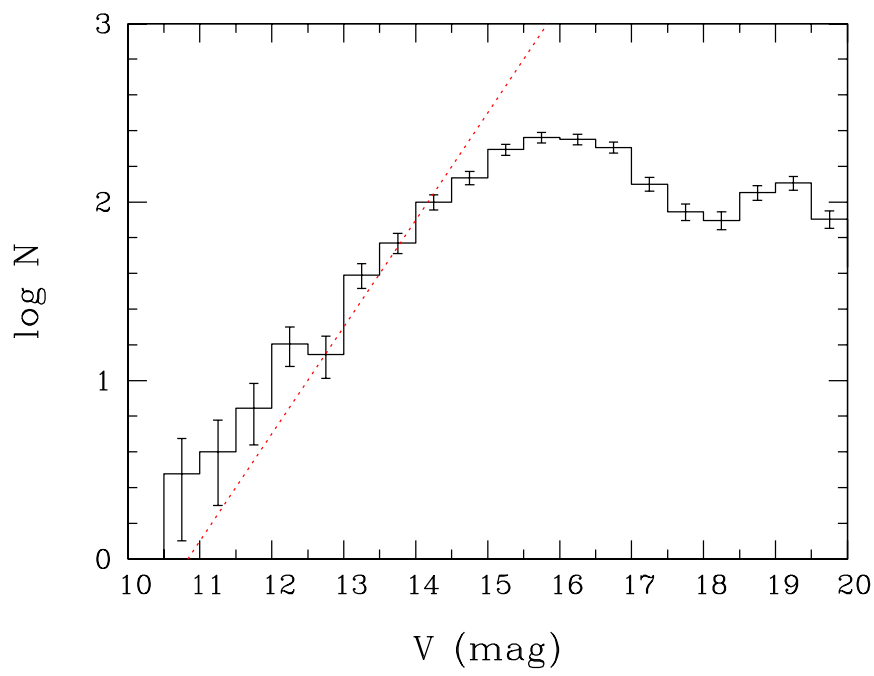

Fig. 9. Logarithm of the number of stars in the WDC as a function of their observed visual magnitude, in bins of $0.5 \mathrm{mag}$. The error bars are from number statistics. A linear relation assuming a volume complete sample (dotted red line) as been fitted to the data in the range $12.5<V<14.5$.

as a function of magnitude. Hence, we predict that only 0.3 field white dwarfs, on average, will be found within $8 \mathrm{~km} \mathrm{~s}^{-1}$ from the $3 \mathrm{D}$ cluster velocity. Furthermore, 3.9 objects, among those 2.4 with $M_{V}<12$ will mimic the 2D velocity within the same range.

Although the relative larger number of white dwarfs at $V<$ 12.5 in Fig. 9 can be explained by statistical fluctuations, they can also indicate that our assumption of a completeness of $100 \%$ up to $V=14.5$ is rather optimistic ${ }^{7}$. A larger completeness correction provides a lower number of field white dwarfs contaminating our sample of Hyades candidates. Therefore the contamination estimates above can be considered as conservative.

\section{Discussion}

Our analysis has so far reviewed the status of the ten classical Hyades white dwarfs and provided improved distances based on the most recent determinations of the atmospheric parameters. Then, we updated the residual velocities with respect to the bulk motion of the cluster. We have furthermore studied 20 new candidates identified in Paper II and in this work. We found that all but one were outside of the tidal radius, and six of them were clearly background objects. There remain 14 objects for which the Hyades membership status is still possible. A comparison of the distribution of the observed candidates versus the simulated field white dwarfs in Figs. 5 and 7 (bottom panel, with the completeness correction) can serve to conclude about the global status of those remaining candidates.

We will first draw our attention on objects that have an age lower than the cluster age (black points in Fig. 5), which are the most logical candidates. There are six such white dwarfs, although this excludes the two binary candidates for which the cooling age is not known (cyan points). Also, the magnetic white dwarf WD $0816+376$ is at 47 pc from the cluster centre according to Table 2, but given the imprecise atmospheric parameters, we can not exclude that it is actually closer (see Table 2). In comparison, the number of contaminants in this age group

\footnotetext{
7 Note that for $V<11$, most objects are white dwarfs in Sirius-like systems, for which the completeness is rather low (Holberg 2009).
}

is 2.4 or 3.3 , including or not the completeness correction, respectively. We find that the probability that six objects are field stars is $4 \%$ or $12 \%$ for a Poisson distribution including or not the completeness correction, respectively. Compared to the field, there is an overdensity of white dwarfs of ages younger than the Hyades. We must remind, however, the difficulty of estimating the uncertainty on the number of contaminants, although we believe that our proposed number is conservative.

If we look closer at the properties of the 6 most promising candidates (identified in Table 2 by their candidate status), we can see from Table 1 that their masses are in the range of $0.66<M_{\odot}<0.98$, with a mean of $0.82 M_{\odot}$, i.e. much higher than the mean mass of field white dwarfs. The probability of 6 field stars having such a high mean mass, assuming the volumecomplete mass distribution of Giammichele et al. (2012), is only $\sim 0.1 \%$. Once again, these 6 objects stand out from the field white dwarfs.

Secondly, we can look at older stars $\left(M_{V}>12\right.$, red points in the Figs. 5 and 7) for which we have four candidates. For the time being, No. 15 remains an additional candidate, given its uncertain nature and imprecise atmospheric parameters. From our simulations, we expect between 1.5 and 26 old objects to contaminate the sample, considering or not the completeness correction, respectively. Since the completeness correction is so high, it is hard to be confident about the expected number of contaminants. Taking literally the mean of 1.5 contaminants, the probability to find 4 is only $7 \%$. However, one would expect that these are the most obvious contaminants, since their ages are inconsistent with the Hyades age.

Nevertheless, an important problem arises if one concludes too fast that the old white dwarfs are contaminants. Indeed, two of these candidates have radial velocities that are in agreement with the Hyades motion (WD 0120-024; Silvestri et al. 2001, and WD 0433+270; Zuckerman et al. 2003), which would make them strong candidates if it was not for their age of about $4 \mathrm{Gyr}$ (see Table 1). These two objects are in common proper motion pairs with main-sequence stars considered as of Hyades origin. We note, however, that Silvestri et al. (2001) also measured a radial velocity for WD $0433+270$, which is incompatible with the value found by Zuckerman et al. (2003), and also incompatible with the radial velocity of its companion, since the mass, hence the gravitational redshift correction, is known for this white dwarf. Our simulations show that we expect to find, on average, 2.3 old objects that mimic the 3D Hyades velocities within $8 \mathrm{~km} \mathrm{~s}^{-1}$, although when accounting for the completeness correction, the number drops to about 0.1 . The fact that we find two of them may be statistically improbable, but not impossible considering low number statistics. If we instead infer that they are not simply field stars that accidentally have the 3D Hyades motion, different scenarios can be conceived. Perhaps the most promising scenario is that these objects could be members of the Hyades supercluster cold kinematic stream which is known to have components that are significantly older than the Hyades (Chereul et al. 1999; Famaey et al. 2007). Other possibilities are that they could have been captured by the Hyades cluster, or they could be younger Fe-core white dwarfs (Catalán et al. 2008), although in the latter case, the authors claim that the white dwarfs would still be too old ( $1 \mathrm{Gyr})$.

As a consequence of the discussion above, further observations are needed to constrain the membership of the Hyades candidates. Accurate trigonometric parallaxes, for instance from Gaia, would provide distances that are independent of $d_{\text {spectro }}$ and therefore can eliminate possible uncertainties due to the atmospheric parameters. This alone, however, is not sufficient to 
conclude about their membership, since their true 3D velocities are not known. Consequently, accurate radial velocity measurements are indispensable to confirm or rule out which of the candidates is of Hyades origin. If consistent with the predicted Hyades radial velocity, the probability of contamination is very low (see Table 3). Due to the extreme broadening of the spectral lines, the spectroscopic measurement of the radial velocity of white dwarfs is extremely difficult to obtain, and only possible with the help of the narrow NLTE cores of $\mathrm{H}_{\alpha}$ (Greenstein et al. 1977; Koester 1987; Falcon et al. 2010). Since this measurement must be corrected for the gravitational redshift, typically $\sim 40 \mathrm{~km} \mathrm{~s}^{-1}$, such a determination is only possible if we have a good knowledge of the mass. For instance, star No. 2 has a radial velocity measurement that is not in agreement with the Hyades motion (see Paper II), even though it is still considered as a classical member. It is more likely, in this case, that the uncertainty of the radial velocity measurement is underestimated. One should therefore be cautious about false negative conclusions from radial velocities alone.

In principle, there is a completely different way to measure the radial velocity astrometrically by determining the perspective acceleration due to the changing distance of the white dwarfs (Oort 1932; Gatewood \& Russell 1974) with Gaia (Anglada-Escudé \& Debes 2010). In this approach no correction for gravitational redshift is needed. Assuming that a $3 \sigma$ deviation of the proper motion during the 5 years mission is required and taking into account that the Gaia proper-motion standard error depends on the magnitude (de Bruijne 2012), one concludes that only bright $(V<13)$ white dwarfs closer than 5 pc allow an estimate of the radial velocity with the needed precision (de Bruijne, priv. comm.). Hence we can not rely on this method for the Hyades.

\subsection{Implications in terms of the Hyades evolution}

This paper has performed a spectroscopic check of the 27 white dwarfs found in Paper II as possible Hyades members. Compared to Paper II, we tried to reveal more kinematic candidates by softening the velocity criterion. Nevertheless, no more than 10 white dwarfs have been found within the presentday tidal radius $(9 \mathrm{pc})$ of the cluster, including one new candidate (No. 20). Also, no objects with an age between 340 and $600 \mathrm{Myr}$ are recovered in this radius range. Here we claim that no more single white dwarfs of ages lower than the Hyades age will be detected within the tidal radius. Outside the tidal radius and up to $40 \mathrm{pc}$, between 6 and 9 white dwarfs with a possible Hyades origin are found. Among those, about three are expected to be contaminants, although a few hot degenerate candidates could also be missing due to the incompleteness of the white dwarf catalogues. Even among the 6 most promising new candidates, all have an age lower than $340 \mathrm{Myr}$, except for No. 26, with an age of 410 Myr. In summary, we face again the long-standing problem (Tinsley 1974; Weidemann 1977) that not enough old white dwarfs are associated with the cluster in comparison to predictions, in particular compared to the most recent $N$-body Hyades cluster simulations of Ernst et al. (2011).

The simulations of Ernst et al. (2011) use the present-day mass distribution of stars within 9 pc of the cluster centre from Paper I among inputs (see their Table 1) to simulate the formation and evolution of the cluster. Their best fit simulations ultimately predict the number, mass, and age of white dwarf members. They forecast that 73 remnants were formed in the cluster so far, including five black holes, 13 neutron stars and 55 white dwarfs. However, many of these remnants have long ago evaporated from the cluster. In the simulations, the newly formed white dwarfs get a kick drawn from a Maxwellian distribution with a $1 \mathrm{D}$ velocity dispersion of $5 \mathrm{~km} \mathrm{~s}^{-1}$, corresponding to a $3 \mathrm{D}$ kick with a mean of $\sim 8 \mathrm{~km} \mathrm{~s}^{-1}$. The present-day consequence of these kicks is that $40 \%$ (22 objects) of the white dwarfs remain within the present-day tidal radius and are dynamically cooled down to show a velocity dispersion of only $\sim 0.5 \mathrm{~km} \mathrm{~s}^{-1}$. It is worth noting that only $\sim 10$ among these bound white dwarfs have ages smaller than 340 Myr (Ernst et al., priv. comm.). On the other hand, the kicks have removed 33 white dwarfs from the cluster. At present, five lie in the range $9<d_{\text {centre }}<30 \mathrm{pc}$, and others are more than $30 \mathrm{pc}$ away from the centre. The authors are cautious to warn that the final number of tidally bound white dwarfs depends critically on the kick velocity. However, there are actually few observational constraints to confirm if white dwarfs obtain such kicks from asymmetrical mass loss in the planetary nebula ejection, and how strong it would be (Davis et al. 2008; Fregeau et al. 2009). We also note that possible encounters with giant molecular clouds or spiral arms are neglected in the simulations.

Compared to the $N$-body simulations of Ernst et al. (2011), the observations cannot account for at least half of the predicted white dwarfs within the tidal radius, but observations and simulations agree for the number of young $(<340 \mathrm{Myr})$ white dwarfs there. It is also worth to note that the simulations predict only a small number (five) of white dwarfs between 9 and $30 \mathrm{pc}$, which would be consistent with our findings of candidates in the $40 \mathrm{pc}$ range. On the other hand, the observations and simulations fully disagree for the older white dwarfs: the simulations suggest at least ten older (>340 Myr) white dwarfs within the tidal radius, but we do not observe any of them. It is very unlikely that the ages of white dwarfs drawn from the cooling sequences of Wood (1995); Fontaine et al. (2001) are significantly underestimated given the consistency between white dwarf cooling ages and main sequence ages in the Hyades and other clusters (De Gennaro et al. 2009; Jeffery et al. 2011). We have verified that even with the calculations of Renedo et al. (2010), which are done by evolving white dwarf progenitors directly from the zeroage main sequence, the ages of the white dwarfs discussed in this work are only changed by $\sim 10 \%$. There are different other explanations for this discrepancy and we review some of them in turn.

One possibility is that more white dwarfs than expected were kicked out of the cluster, and that most of them are now far from the centre. The kick velocities added to white dwarfs in the Ernst et al. (2011) simulations can be seen as some kind of free parameter. However, since objects within the tidal radius thermally relax on short time scales, they can not be used to constrain this parameter. The observed spatial density and residual velocities of white dwarfs outside of the tidal radius could in principle be used, but these numbers are poorly constrained by the current work. We suggest that new simulations could be run with a wide number of assumptions on the kick velocities to predict how the number density and residual velocities would be modified. Fellhauer et al. (2003) suggest that older white dwarfs are preferentially lost due to their higher recoil speed, which is in turn due to the larger amount of mass loss of their high-mass progenitors, although it only remains an hypothesis. We note that similar simulations for the white dwarfs in other open clusters could be very helpful to constrain the distribution of kick velocities.

Another scenario is that fewer white dwarfs than predicted were ever formed in the Hyades, which would correspond to a change of the Initial Mass Function (IMF) at its high-mass end. Dobbie et al. (2006) estimate from their initial vs. final mass relation that the classical white dwarfs come from main-sequence 
progenitors in the range $\sim 2.5-4 M_{\odot}$. Therefore, to obtain more promising predictions from the simulations, one would have to modify the formation rate of white dwarfs for main-sequence stars with $M>4 M_{\odot}$. Related to this, the fact that initial vs. final mass relation for white dwarfs is not so well constrained for high masses (Dobbie et al. 2006, see their Fig. 3) might also have an effect on the number of white dwarfs vs. neutron stars and the age distribution of the remnants. Having a steeper initial mass function in this regime could have a strong effect on the predicted number of white dwarfs (Böhm-Vitense 2007). In this context we note that the present-day mass function of the Hyades derived in Paper I (Fig. 10) tolerates a steeper slope leading to less white dwarf progenitors.

Thirdly, the topic of white dwarfs in binary systems has only been touched marginally in this paper. Holberg (2009) claims that $32 \pm 8 \%$ of the local white dwarfs are in binary systems. Among the classical members, we find that three out of ten are binaries, a number similar to field objects. However, it would be dangerous to extrapolate that the fraction of binaries is the same for field white dwarfs and the Hyades. Sollima et al. (2010) estimate that the binary fraction of stars in open clusters is between 35 and $70 \%$. Based on the Hipparcos data from Perryman et al. (1998), Böhm-Vitense (2007) found that the binary fraction in the Hyades increases from $30 \%$ for late-G to $88 \%$ for earlyA stars. While we claimed previously that virtually all single white dwarfs have been discovered in the cluster centre area, it is likely not the case for white dwarfs in unresolved binaries. For instance, V471 Tau and HD 27483 (Nos. 1 and 4) have not been discovered by systematic surveys, but from specific studies of Hyades main-sequence members (Nelson \& Young 1970; Böhm-Vitense 1993). Since old and massive Hyades white dwarfs (e.g. with masses of $\sim 0.8 M_{\odot}$ ) are completely hidden in the visible by their main-sequence companion in the majority of cases, they can only be detected by UV observations of each main-sequence member. However, Böhm-Vitense (1995) found no white dwarf companion in a sample of IUE spectroscopic observations for 27 Hyades F stars. Furthermore, Paulson et al. (2004) found no evidence of white dwarf companions in a radial velocity survey of 94 Hyades stars. All in all, there is so far no observational evidence that the missing white dwarfs older than $\sim 340$ Myr are hidden in binary systems.

\section{Summary}

The starting point of our work has been the Hyades white dwarf candidates kinematically and photometrically selected by Schilbach \& Röser (2012) from the PPMXL survey. We evaluated the membership status of these candidates by using spectroscopic and photometric observations, from which we determined new distances of the white dwarfs from the Sun and from the cluster centre as well as residual velocities with respect to the Hyades bulk motion. We find a very good agreement between kinematic and spectroscopic distance predictions for the 10 classical white dwarf members, confirming that the kinematic and photometric data sets, as well as model atmospheres that we rely on, are consistent with each others.

We ruled out 6 of the Hyades candidates from Paper II as background stars, but also added 3 new objects by increasing the residual velocity upper limit for membership selection to $8 \mathrm{~km} \mathrm{~s}^{-1}$. There remain 14 candidates, five of which have cooling ages higher than the age of the cluster. We do not consider them as of Hyades origin. For another three, the atmospheric parameters are rather imprecise or could not be determined, leaving us with six spectroscopically confirmed hot and young white dwarfs consistent with a Hyades membership. To estimate the contamination fraction from field white dwarfs, we performed Monte Carlo simulations. The result is that $\sim 2-3$ hot and young white dwarfs may contaminate our sample yielding that the probability that all six are contaminants is low. A final confirmation of the candidates needs accurate radial velocity measurements properly corrected for gravitational redshift.

The major discrepancy between observations of white dwarfs of Hyades origin and the predictions by simulations is the lack of about a dozen white dwarfs with cooling ages between 340 and $~ 600 \mathrm{Myr}$, which should be found within the present-day tidal radius ( $\sim 9 \mathrm{pc})$ of the cluster. Possible explanations for this are: a) at their formation, within the first $300 \mathrm{Myr}$ of the cluster's life, white dwarfs get a large $\left(\gtrsim 5 \mathrm{~km} \mathrm{~s}^{-1}\right)$ kick velocity relative to the cluster motion which sweeps them farther out than $40 \mathrm{pc}$ from the centre; b) the high-mass end of the IMF is steeper than the Salpeter slope of 2.3 assumed in the simulations, hence leading to a lesser number of massive stars; and c) there is still a number of so-far unrevealed white dwarfs hidden in binary systems. There seems to be no way, at present, to decide which of these scenarios is the dominant one in consistence with the conclusion of Williams (2004).

Acknowledgements. We wish to dedicate this work to the memory of Volker Weidemann who was very fond of the Hyades white dwarfs. P.-E. T. is supported by the Alexander von Humboldt Foundation. This work was partially supported by Sonderforschungsbereich SFB 881 "The Milky Way System" (Subproject A4 and B5) of the German Research Foundation (DFG).

\section{References}

Angel, J. R. P., Borra, E. F., \& Landstreet, J. D. 1981, ApJS, 45, 457 Anglada-Escudé, G., \& Debes, J. 2010, in IAU Symp. 261, eds. S. A. Klioner,

P. K. Seidelmann, \& M. H. Soffel (Cambridge: CUP), 342

Bergeron, P., Saffer, R. A., \& Liebert, J. 1992, ApJ, 394, 228

Bergeron, P., Leggett, S. K., \& Ruiz, M. T. 2001, ApJS, 133, 413

Bergeron, P., Gianninas, A., \& Boudreault, S. 2007, in Proc. 15th European Workshop on White Dwarfs, eds. R. Napiwotzki, \& M. Burleigh (San Francisco: ASP), 372, 29

Bergeron, P., Wesemael, F., Dufour, P., et al. 2011, ApJ, 737, 28

Böhm-Vitense, E. 1993, AJ, 106, 1113

Böhm-Vitense, E. 1995, AJ, 110, 228

Böhm-Vitense, E. 2007, AJ, 133, 1903

Burleigh, M. R., Barstow, M. A., \& Holberg, J. B. 1998, MNRAS, 300, 511

Catalán, S., Ribas, I., Isern, J., \& García-Berro, E. 2008, A\&A, 477, 901

Chereul, E., Crézé, M., \& Bienaymé, O. 1999, A\&AS, 135, 5

Copenhagen Univ. Obs., Inst. of Astronomy, Cambridge, UK, \& Real Instituto

y Observatorio de La Armada, San Fernando 2006, VizieR Online Data Catalog, I/304

Davis, D. S., Richer, H. B., King, I. R., et al. 2008, MNRAS, 383, L20

de Bruijne, J. H. J. 2012, Ap\&SS, 341, 31

De Gennaro, S., von Hippel, T., Jefferys, W. H., et al. 2009, ApJ, 696, 12

Dobbie, P. D., Napiwotzki, R., Burleigh, M. R., et al. 2006, MNRAS, 369, 383

Dobbie, P. D., Napiwotzki, R., Burleigh, M. R., et al. 2009, MNRAS, 395, 2248

Eggen, O. J. 1993, AJ, 106, 642

Eisenstein, D. J., Liebert, J., Harris, H. C., et al. 2006, ApJS, 167, 40

Ernst, A., Just, A., Berczik, P., \& Olczak, C. 2011, A\&A, 536, A64

Falcon, R. E., Winget, D. E., Montgomery, M. H., \& Williams, K. A. 2010, ApJ, 712,585

Famaey, B., Pont, F., Luri, X., et al. 2007, A\&A, 461, 957

Fellhauer, M., Lin, D. N. C., Bolte, M., et al. 2003, ApJ, 595, L53

Fontaine, G., Brassard, P., \& Bergeron, P. 2001, PASP, 113, 409

Fregeau, J. M., Richer, H. B., Rasio, F. A., \& Hurley, J. R. 2009, ApJ, 695, L20 Gatewood, G., \& Russell, J. 1974, AJ, 79, 815

Giammichele, N., Bergeron, P., \& Dufour, P. 2012, ApJS, 199, 29

Gianninas, A., Bergeron, P., \& Ruiz, M. T. 2011, ApJ, 743, 138

Greenstein, J. L., Boksenberg, A., Carswell, R., \& Shortridge, K. 1977, ApJ, 212,186

Harris, H. C., Munn, J. A., Kilic, M., et al. 2006, AJ, 131, 571

Holberg, J. B. 2009, J. Phys. Conf. Ser., 172, 012022

Holberg, J. B., \& Bergeron, P. 2006, AJ, 132, 1221 
P.-E. Tremblay et al.: Spectroscopic and photometric studies of white dwarfs in the Hyades

Holberg, J. B., Bergeron, P., \& Gianninas, A. 2008, AJ, 135, 1239 Jeffery, E. J., von Hippel, T., DeGennaro, S., et al. 2011, ApJ, 730, 35 Jordan, S. 1993, in White Dwarfs: Advances in Observation and Theory, ed. M. A. Barstow (Dordrecht: Kluwer), 403, 333

Kalirai, J. S., Richer, H. B., Fahlman, G. G., et al. 2001a, AJ, 122, 266

Kalirai, J. S., Ventura, P., Richer, H. B., et al. 2001b, AJ, 122, 3239

Kalirai, J. S., Fahlman, G. G., Richer, H. B., \& Ventura, P. 2003, AJ, 126, 1402

Koester, D. 1987, ApJ, 322, 852

Koester, D., Kepler, S. O., Kleinman, S. J., \& Nitta, A. 2009a, J. Phys.: Conf. Ser., 172, 012006

Koester, D., Voss, B., Napiwotzki, R., et al. 2009b, A\&A, 505, 441

Kowalski, P. M., \& Saumon, D. 2006, ApJ, 651, L137

Liebert, J., Bergeron, P., \& Holberg, J. B. 2005, ApJS, 156, 47

Limoges, M.-M., \& Bergeron, P. 2010, ApJ, 714, 1037

Luyten, W. J. 1977, White Dwarfs II (Minneapolis: Univ. of Minnesota Press)

McCook, G. P., \& Sion, E. M. 1999, ApJS, 121, 1

Nelson, B., \& Young, A. 1970, PASP, 82, 699

Oort, J. H. 1932, Bull. Astron. Inst. Neth, 238, 287

Paulson, D. B., Cochran, W. D., \& Hatzes, A. P. 2004, AJ, 127, 3579

Pels, G., Oort, J. H., \& Pels-Kluyver, H. A. 1975, A\&A, 43, 423

Perryman, M. A. C., Brown, A. G. A., Lebreton, Y., et al. 1998, A\&A, 331, 81

Portegies Zwart, S. F., McMillan, S. L. W., Hut, P., \& Makino, J. 2001, MNRAS, 321, 199

Renedo, I., Althaus, L. G., Miller Bertolami, M. M., et al. 2010, ApJ, 717, 183

Richer, H. B., Fahlman, G. G., Rosvick, J., \& Ibata, R. 1998, ApJ, 504, L91

Röser, S., Demleitner, M., \& Schilbach, E. 2010, AJ, 139, 2440

Röser, S., Schilbach, E., Piskunov, A. E., Kharchenko, N. V., \& Scholz, R.-D. 2011, A\&A, 531, A92

Rubin, K. H. R., Williams, K. A., Bolte, M., \& Koester, D. 2008, AJ, 135, 2163

Schilbach, E., \& Röser, S. 2012, A\&A, 537, A129

Schulz, H., \& Wegner, G. 1981, A\&A, 94, 272
Silvestri, N. M., Oswalt, T. D., Wood, M. A., et al. 2001, AJ, 121, 503

Silvestri, N. M., Hawley, S. L., \& Oswalt, T. D. 2005, AJ, 129, 2428

Sion, E. M., Holberg, J. B., Oswalt, T. D., McCook, G. P., \& Wasatonic, R. 2009, AJ, 138, 1681

Sion, E. M., Bond, H. E., Lindler, D., et al. 2012, ApJ, 751, 66

Smith, J. A. 1997, Ph.D. Thesis, Florida Institute of Technology

Sollima, A., Carballo-Bello, J. A., Beccari, G., et al. 2010, MNRAS, 401, 577

Tinsley, B. M. 1974, PASP, 86, 554

Tremblay, P.-E., \& Bergeron, P. 2007, ApJ, 657, 1013

Tremblay, P.-E., \& Bergeron, P. 2008, ApJ, 672, 1144

Tremblay, P.-E., \& Bergeron, P. 2009, ApJ, 696, 1755

Tremblay, P.-E., Bergeron, P., \& Gianninas, A. 2011a, ApJ, 730, 128

Tremblay, P.-E., Ludwig, H.-G., Steffen, M., Bergeron, P., \& Freytag, B. 2011b, A\&A, 531, L19

van den Heuvel, E. P. J. 1975, ApJ, 196, L121

van Leeuwen, F. 2009, A\&A, 497, 209

Vanlandingham, K. M., Schmidt, G. D., Eisenstein, D. J., et al. 2005, AJ, 130, 734

Vennes, S., Chayer, P., Dupuis, J., \& Lanz, T. 2005, in 14th European Workshop on White Dwarfs, eds. D. Koester, \& S. Moehler (San Francisco: ASP), ASP Conf. Ser., 334, 185

Weidemann, V. 1977, A\&A, 59, 411

Weidemann, V., \& Koester, D. 1980, A\&A, 85, 208

Weidemann, V., Jordan, S., Iben, I., Jr., \& Casertano, S. 1992, AJ, 104, 1876

Williams, K. A. 2004, ApJ, 601, 1067

Williams, K. A., \& Bolte, M. 2007, AJ, 133, 1490

Williams, K. A., Bolte, M., \& Koester, D. 2009, ApJ, 693, 355

Wood, M. A. 1995, in White Dwarfs: Proc. 9th European Workshop on White Dwarfs, eds. D. Koester, \& K. Werner (Berlin: Springer), 41

Zacharias, N., Finch, C., Girard, T., et al. 2010, AJ, 139, 2184

Zuckerman, B., Koester, D., Reid, I. N., \& Hünsch, M. 2003, ApJ, 596, 477 